\title{
Anogenital mammary-like gland adenoma: report of two cases and review of literature
}

\begin{abstract}
Hidradenoma papilliferum also known as papillary hidradenoma is a benign, slow- growing, asymptomatic, adnexal tumor that primarily affects the anogenital area of adult women. The origin of hidradenoma papilliferum was previously thought to be an apocrine sweat gland; recent studies have suggested that it may derive from the anogenital mammarylike gland. It usually presents as slow-growing, asymptomatic flesh-colored nodule in the anogenital area. Its dermoscopic features are poorly reported in the literature. The nodule is composed of tubules and papillary structures arranged in a frond-like pattern. Treatment of hidradenoma papilliferum consists of complete surgical excision.
\end{abstract}

We report the characteristics of two cases of this rare entity.

Keywords: hidradenoma papilliferum, anogenital mammary- like glands, vulva
Volume 9 Issue 5 - 2018

Ouiame EL Jouari,' Anas Zaougui, ${ }^{2}$ Jihane Ziani,' Ghita Senhaji,' Salim Gallouj,' Moulay Hassan Farih, ${ }^{2}$ Fatima Zahra Mernissi'

'Department of Dermatology, University Hospital Hassan II, Morocco

${ }^{2}$ Department of Urology, University Hospital Hassan II, Morocco

Correspondence: Ouiame EL Jouari, Department of Dermatology, University Hospital Hassan II, Road Sidi Hrazem, Fez, Morocco, Tel 212645768798,

Email eljouariouiame@gmail.com

\section{Introduction}

Hidradenoma papilliferum (HP) is a rare benign tumor affects vulvar, perineal and perianal skin of adult women. It usually presents as slow-growing, asymptomatic flesh-colored nodule in the anogenital area. The nodule is composed of tubules and papillary structures arranged in a frond-like pattern. We aim to discuss clinical, dermoscopical aspects and histological features of this tumor and surgical modalities. We also emphasize the importance of keeping this tumor in mind among the possibilities of differential diagnosis of anogenital area nodules.

\section{Cases presentation}

Case N1: A 24-year-old woman with 2 years' history of a small painless vulval nodule which increased in size, what motivated the patient to consult in our department. The dermatological examination revealed a $7 \mathrm{~mm}$ pink papule, firm in consistency, located on the right lip of the labium majora (Figure 1). Dermoscopy of the lesion revealed pink and white structures with dotted vessels (Figure 2).

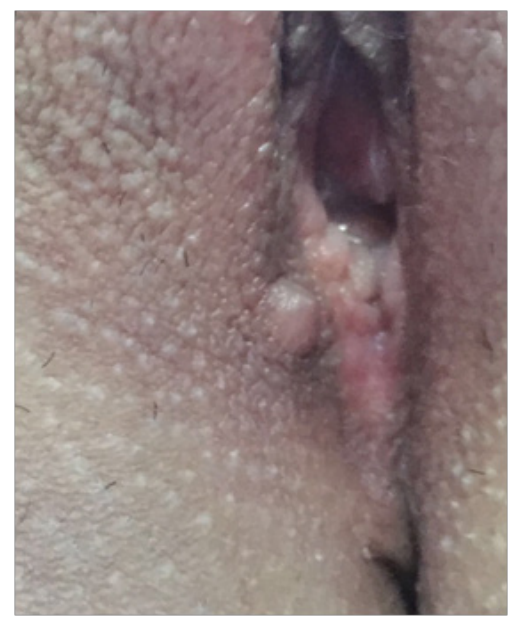

Figure I a $7 \mathrm{~mm}$ pink papule, firm in consistency, located on the right lip of the labium majora.

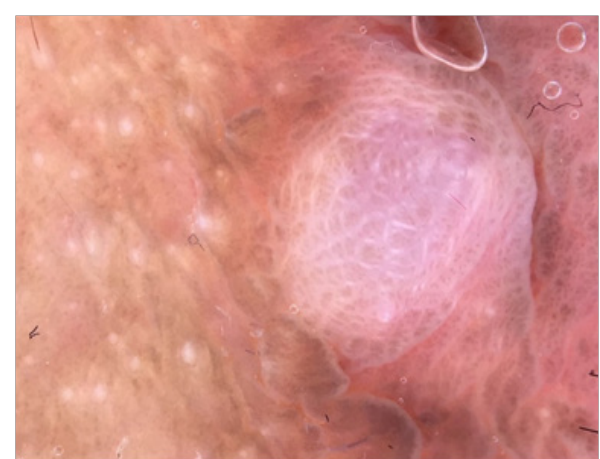

Figure 2 pink and white structures with dotted vessels.

Case N2: A 36-year-old woman with three years' history of kidney stones. She was referred by her urologist for management of a nodule of the vulvar region wich was finding of a physical examination. Dermatological examination revealed a skin-colored nodule of $8 \mathrm{~mm}$ in size, firm in consistency with a sessile base on the left lip of the labium majora (Figure 3). No local or regional lymph-node was palpable. The dermoscopy had objective an erythematous background with multiple fine telangiectasia (Figure 4).

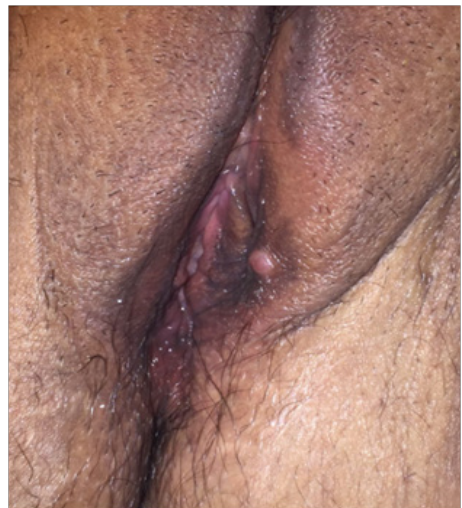

Figure 3 a skin-colored nodule of $8 \mathrm{~mm}$ in size, firm in consistency with a sessile base on the left lip of the labium. majora. 
The nodules were surgically excised. Histopathology demonstrated an epithelial proliferation of tubular and acinar structures coated by a double layer of abundant ductal cells and myoepithelial cells on the outer layer (Figure 5). The current decline is 18 and 12 months respectively without recurrence.

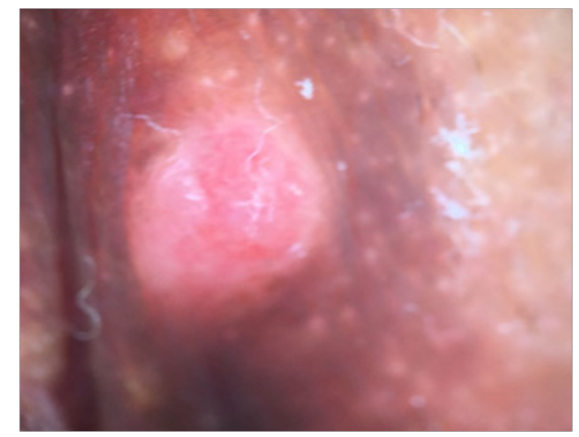

Figure 4 an erythematous background with multiple fine telangiectasia.

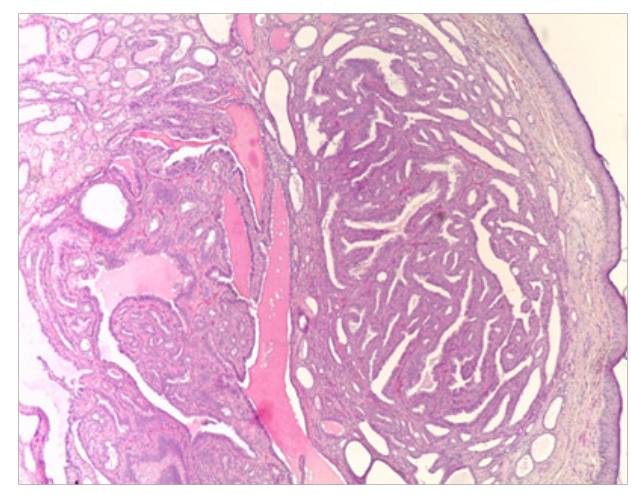

Figure 5 Histopathology showing an epithelial proliferation of tubular and acinar structures coated by a double layer of abundant ductal cells and myoepithelial cells on the outer layer $(\mathrm{H}$ and $\mathrm{E}, \times 100)$.

\section{Discussion}

Here we present two rare case of a histologically confirmed uncommun tumors of anogenital area and we detail their characteristics which are lacking in the literature. Hidradenoma papilliferum also known as papillary hidradenoma is a benign, slow- growing, asymptomatic, adnexal tumor that primarily affects the anogenital area of adult women. ${ }^{1}$ It was first described in 1878 by Werth. Since that time, different authors have suggested various theories relating to pathogenesis and origins of this tumor. ${ }^{2}$ Although the origin of HP was previously thought to be an apocrine sweat gland, recent studies have suggested that it may derive from the anogenital mammarylike gland. ${ }^{3}$ Clinically, the majority of hidradenoma papilliferums are usually present as a solitary, asymptomatic, well-circumscribed, slowly growing, nodular, nonulcerated, cystic or pedunculated lesion measuring in most cases between 0.5 and $3 \mathrm{~cm}$ in diameter. ${ }^{1}$ The overlying skin may be smooth, thickened, atrophic or ulcerated, and may have a skin-coloured, red, brown or blue appearance. Some tumors may spontaneously drain serous or haemorrhagic material. ${ }^{4}$ This neoplasm may be found on the labia minora (50\%) labia majora $(40 \%)$, fourchette $(7 \%)$ and clitoris $(3 \%) .^{5}$ Rarely, it can be seen over the head and neck, and over modified apocrine gland areas such as breast, external ear canal, and eyelid. It is then referred to as ectopic hidradenoma papilliferum. ${ }^{6}$ Due to its variable and nonspecific clinical presentation it might be difficult to make a correct diagnosis of this tumor. ${ }^{4}$ In our cases, the tumors were asymptomatic and diagnosis was finding of a physical examination, suggesting an underestimation of the prevalence of these tumors. Its differential diagnosis includes various adnexal tumors, epidermal cysts, cutaneous metastases, basal cell carcinoma and melanoma. ${ }^{4}$ This makes biopsy of all vulvar lesions necessary in order to establish a diagnosis and rule out malignancy. ${ }^{5}$ The most common dermoscopic pattern of hidradenoma papilliferum is homogeneous area which occupies the whole lesion with vascular and white structures is the most common dermoscopic pattern. The color of this total homogeneous area is pinkish in non-pigmented hidradenoma, bluish, or less commonly brownish in pigmented hidradenoma. The vascular structures objective is arborizing telangiectasias, polymorphous atypical vessels. ${ }^{4}$ For both patients, the same dermoscopic aspects were revealed. Histopathological examination revealed a partly solid or solid-cystic dermal lesion with anastomosing papillary structures and tubules as well as some glandular areas. The epithelial lining consists of an inner layer of columnar cells with decapitation secretion in the lumen and frequently an outer myoepithelial layer. ${ }^{3,6}$ Treatment of hidradenoma papilliferum consists of complete surgical excision of the tumor with free margins. Recurrence has been reported but is uncommon. ${ }^{5}$ In our case, the direct approach was sufficient for complete excision because the lesion was located in the labium majora. The tumor has got a good prognosis and malignant transformation is extremely rare. ${ }^{6}$

\section{Conclusion}

The adenoma of the ano-genital mammary glands is a benign glandular tumor. Its clinical aspects are varied but its locations are very characteristic. Moreover, His histological diagnosis is easy and reassuring. Simple excision is almost always curative. When an adult female presents with a nodular lesion in the anogenital area, hidradenoma papilliferum should be kept in mind along with other differentials diagnosis such as epidermoid cysts, sexually transmitted diseases, melanoma, squamous cell or basal cell carcinoma.

\section{Acknowledgements}

None.

\section{Conflict of interest}

The authors declare that they have no conflict of interest.

\section{References}

1. El-Khoury J, Renald MH, Plantier F, et al. Vulvar hidradenoma papilliferum (HP) is located on the sites of mammary-like anogenital glands (MLAGs): Analysis of the photographs of 52 tumors. J Am Acad Dermatol. 2016;75(2):380-384.

2. Konstantinova AM, Michal M, Kacerovska D, et al. Hidradenoma Papilliferum: A Clinicopathologic Study of 264 Tumors From 261 Patients, With Emphasis on Mammary-Type Alterations. Am J Dermatopathol. 2016;38(8):598-607.

3. Kurashige Y, Kishida K, Kurashige K, et al. Hidradenoma papilliferum of the vulva in association with an anogenital mammary-like gland. $J$ Dermatol. 2014;41(5):411-413.

4. Serrano P, Lallas A, Del Pozo LJ, et al. Dermoscopy of Nodular Hidradenoma, a Great Masquerader: A Morphological Study of 28 Cases. Dermatology. 2016;232(1):78-82.

5. Hernández-Angeles C, Nadal A, Castelo-Branco C. Hidradenoma papilliferum of the vulva in a postpartum woman: A case report. J Obstet Gynaecol. 2017;37(5):683-684.

6. Kambil SM, Bhat RM, D'Souza DC. Hidradenoma papilliferum of the vulva. Indian Dermatol Online J. 2014;5(4):523-524. 